\title{
Microbiological quality of broiler carcasses during slaughter processing
}

\author{
Irena Svobodová1, Gabriela Bořilová1, Radka Hulánková1, Iva Steinhauserová ${ }^{2}$ \\ ${ }^{1}$ Department of Meat Hygiene and Technology, Faculty of Veterinary Hygiene and Ecology, \\ University of Veterinary and Pharmaceutical Sciences Brno, Czech Republic \\ ${ }^{2}$ CEITEC - Central European Institute of Technology, University of Veterinary and Pharmaceutical \\ Sciences Brno, Czech Republic
}

Received August 29, 2011

Accepted January 18, 2012

\begin{abstract}
Microbial contamination of poultry carcasses can be influenced by many factors during transport and slaughtering. The aim of this study was to evaluate the impact of four processing steps (plucking, evisceration, washing and chilling) on the total viable counts (TVC), counts of Escherichia coli, Salmonella spp. and Listeria spp. incidence on broiler carcasses. A total of 160 broiler carcasses originating from one farm were collected during one year period at a Czech slaughterhouse and examined. Both TVC and E. coli counts decreased during processing from $4.6 \log \mathrm{cfu} / \mathrm{cm}^{2}$ and $3.5 \log \mathrm{cfu} / \mathrm{cm}^{2}$ to $3.7 \log \mathrm{cfu} / \mathrm{cm}^{2}$ and $1.8 \log \mathrm{cfu} / \mathrm{cm}^{2}$, respectively, with a major impact of washing on TVC and washing and chilling on E. coli decrease $(P<0.001)$. Both Salmonella spp. (6 strains) and Listeria spp. (12 strains, none of $L$. monocytogenes) were found sporadically in all processing steps followed. However, a decreasing trend was observed in Salmonella counts and Listeria spp. incidence during the processing. Thus, this study brings new valuable information on the dynamics of microflora during modern poultry processing.
\end{abstract}

Poultry carcass, plucking, evisceration, chilling, microbial contamination, Escherichia coli, Listeria, Salmonella

Over the past 20 years, poultry meat production and consumption worldwide has increased very rapidly. This has led to intensive animal production with an increase in both the number of farms and in flock size. Broilers are normally raised on litter floors and this may lead to contamination of poultry both with spoilage microorganisms and also with human pathogens, such as Salmonella spp., Campylobacter spp., Clostridium perfringens, Listeria monocytogenes and Escherichia coli or Staphylococcus aureus. Young animals show symptoms of bacterial infection only occasionally but most of them are healthy carriers of pathogens and they are not excluded from farm or from slaughter during ante mortem inspection. Epidemiological data suggest that contaminated products of animal origin, especially poultry, contribute significantly to foodborne diseases. Reduction of raw poultry contamination levels would thus have a large impact on reducing the incidence of illness (Keener et al. 2004).

Transport and slaughter of poultry involve a number of operations that may substantially affect the extent of poultry contamination. Due to stress during transport, excretion patterns of birds carrying e.g. Salmonella can change through disturbance of intestinal function or even damage to the birds' intestinal tract to such extent that they may adversely affect their immune system (Cox and Pavic 2010).

An important process operation that impacts the presence of microorganisms in poultry slaughter is scalding. At present, the trend is to scald poultry at lower temperatures $\left(50-52{ }^{\circ} \mathrm{C}\right)$, which are more suitable for air-chilled poultry. Lower scalding temperatures may, however, allow some microorganisms including pathogens to survive. A way of avoiding this problem is to use multistage scalding, where poultry is scalded in several 
scald tanks lined one behind another, which substantially reduces contamination on poultry surfaces (Berrang et al. 2008).

The next process operation is plucking, which is closely related to the scalding operation. The main hygienic problem is cross-contamination via equipment or via aerosols in the air.

Evisceration is the first stage of the clean part of the slaughter process. Consisting of several stages, evisceration starts with head removal followed by opening of the body cavity, removal of intestines, and ends with the cleaning of the carcass (Cox and Pavic 2010). From the hygienic point of view, attention is paid to the removal of the intestines and the prevention of cross-contamination with faecal material.

The next processing step is chilling which is essential to control microbial growth (James et al. 2006). Common methods include continuous mechanical immersion, chilling and airblast chilling, with or without the incorporation of water-sprays to maintain product yield and enhance cooling by evaporation (Mead 2004).

It follows from the above overview of basic processing steps in broiler slaughter that there are many steps in the poultry meat processing that could significantly influence the extent of poultry contamination and thus also its marketability and incidence of pathogenic microorganisms. The most critical processing steps in this respect include scalding, plucking, evisceration and the type of poultry chilling (Keener et al. 2004).

The aim of this study was to evaluate the impact of four selected processing steps (plucking, evisceration, washing and chilling) on the dynamics of the total viable count (TVC), and counts of E. coli, Salmonella spp. and Listeria spp.

\section{Materials and Methods}

\section{Sampling}

Samples of whole carcasses were obtained from a broiler processing plant during a one year period. The samples were collected eight times at regular 6-week intervals. All broilers came from one intensive poultry farm; using the all-in-all-out production system, the farm had a production capacity of 280,000 broilers. The average rearing period was 36 days (33-39 days), the average weight was $2.1 \mathrm{~kg}$ and the mortality on the farm was $3.6 \%$. Broilers of Ross 308 and Cobb 500 hybrid lines were fed with a commercial feed mix with coccidiostats (nicarbazin and monensin), but without growth-promoting antibiotics. The farm was located $60 \mathrm{~km}$ from the slaughterhouse, so the journey duration was short in all cases.

The poultry slaughter process was typical for the industry. It consisted of slaughtering, scalding at $54 \pm 2{ }^{\circ} \mathrm{C}$ for $180 \mathrm{~s}$, plucking, washing, evisceration and evaporative chilling for $70 \mathrm{~min}$. An inside/outside washer was used for cleaning carcasses before chilling. The temperature in the chilling tunnel was less than $0{ }^{\circ} \mathrm{C}$ and the surface temperature of carcasses after chilling was less than $4^{\circ} \mathrm{C}$. The processing line had the capacity of 8,500 carcasses per hour.

The samples were collected from four different points along the processing line. The first sampling point was at the line after plucking (including head removal and hock cutting), the second was after evisceration (including removal of gastrointestinal tract and lungs), the third was after washing before chilling and the last point was after evaporative chilling. Five broiler carcasses from each sampling point were collected $(n=20)$; a total of 160 broiler carcasses were analysed.

Each sample was represented by the whole carcass, which was removed from the processing line. The samples were placed into a sterile plastic bag and sent in an insulated box with refrigerant gel packs to the laboratory.

\footnotetext{
Microbiological analyses
}

For microbiological analyses, the carcass was sampled by the whole carcass rinse (WCR) technique as described by Lindblad et al. (2006). Buffered peptone water (Merck, Germany) was used for sample dilution.

Total viable counts were determined according ISO 4833:2003 standard method using Plate Count Agar (Merck) after incubation for $72 \mathrm{~h}$ at $30{ }^{\circ} \mathrm{C}$. Escherichia coli were determined according ISO 16649-2:2001 standard method using Chromocult (TBX) agar (Merck) after incubation for $24 \mathrm{~h}$ at $44{ }^{\circ} \mathrm{C}$.

The presence of Salmonella spp. was determined according to modified ISO 6579:2003 standard method. As the selective agar media, XLT4 agar and Brilliant Green Agar were used, incubated at $37^{\circ} \mathrm{C}$ for $24 \mathrm{~h}$. After confirmation, isolates were serotyped and phagotyped at the National Institute of Public Health, Brno, Czech Republic. The most probable number (MPN) method was used for the counting of Salmonella spp. All the tubes were prepared in triplicate. Positive tubes were scored and the MPN values were calculated using Thomas' equation (Blodgett 2006). All the media used for the determination of Salmonella spp. were purchased from Oxoid, United Kingdom. 
For the detection of Listeria spp., ISO 11290-1:1999 standard method was used. Confirmation and species identification of pure cultures were performed using PCR (Bubert et al. 1992). Species-specific identification was provided according to protocol described by Huang et al. (2007).

Evaluation of results

The concentrations in carcass rinse $(\mathrm{cfu} / \mathrm{ml})$ were converted to concentrations $\mathrm{cfu} / \mathrm{cm}^{2}$ after the method of Thomas (1978) and Lindblad et al. (2006). Numbers of cfu were $\log _{10}$ transformed. The Lilliefors test was used to determine whether bacterial concentrations (log $\mathrm{cfu} / \mathrm{cm}^{2}$ per carcass) were normally distributed. All quantitative data were statistically evaluated by the analysis of variance (ANOVA) and Tukey HSD Test.

\section{Results}

Total viable counts (TVC) of individual batches ascertained immediately after plucking showed considerable differences and ranged from $3.8 \log$ to $5.6 \log$ (mean $4.6 \log \pm 0.4$ ). After evisceration, TVC values decreased slightly to the average value of $4.4 \log \pm 0.6$; the maximum and minimum values (5.6 log and $3.6 \log$, respectively) were, however, very close to those found after plucking. A more significant decrease occurred after washing where mean TVC value was $4.0 \log \pm 0.8$ (in the range from $2.3 \log$ to $5.9 \log$ ). Evaporative chilling was the next processing step that reduced TVC to the mean value of $3.7 \log \pm 0.6$ (the range from $2.3 \log$ to $5.2 \mathrm{log}$ ). On the basis of statistical evaluation evisceration did not influence TVC values $(P<0.348)$ so much as washing or chilling $(P<0.179)$. Significant differences were found between TVC after plucking and after chilling $(P<0.001)$, and particularly between TVC levels after plucking and after washing $(P<0.001)$ (Fig. 1). It follows from the above that the decisive processing step from the TVC level's point of view is washing.

E. coli counts (Fig. 2) dropped with each subsequent processing step just as TVC values. Mean values after plucking and after evisceration were $3.5 \log \pm 0.7$ and $3.1 \log$ \pm 0.7 , respectively; evisceration had no effect on $E$. coli counts $(P<0.317)$. A marked drop occurred after washing, where mean $E$. coli counts were $2.7 \log \pm 0.6$ and then after chilling where they were around $1.8 \log \pm 0.8(P<0.001)$.

Only six of the total of 160 broiler carcasses $(3.75 \%)$ were positive for Salmonella spp. Five isolates were identified as $S$. Montevideo and one as $S$. Enteritidis PT4b. Evaluation of Salmonella counts as log MPN per carcass showed the highest counts after plucking $(2.11 \mathrm{log})$. In each subsequent processing step, the numbers were gradually lower $(1.56 \mathrm{log}$ after evisceration, $<1.53 \mathrm{log}$ after washing and $<1.08 \log$ after chilling).

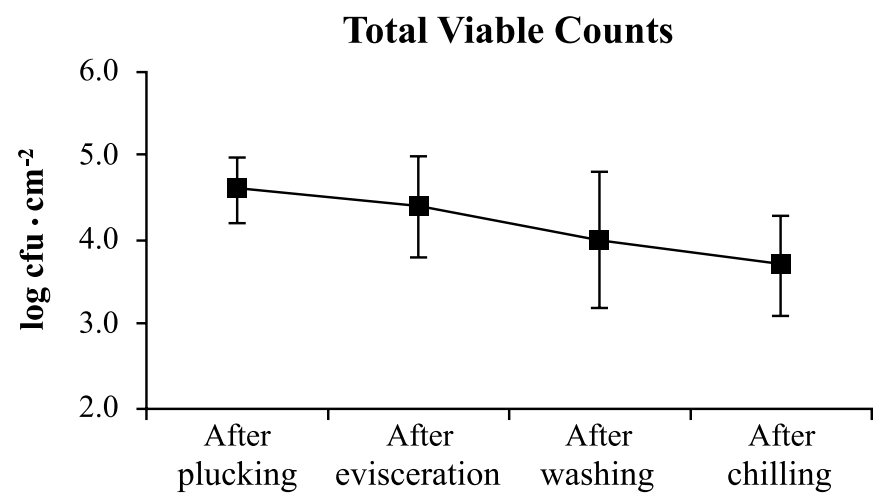

Sampling points

Fig. 1. Total viable counts (mean $\pm \mathrm{SD}$ ) on broiler carcasses during slaughter processing 


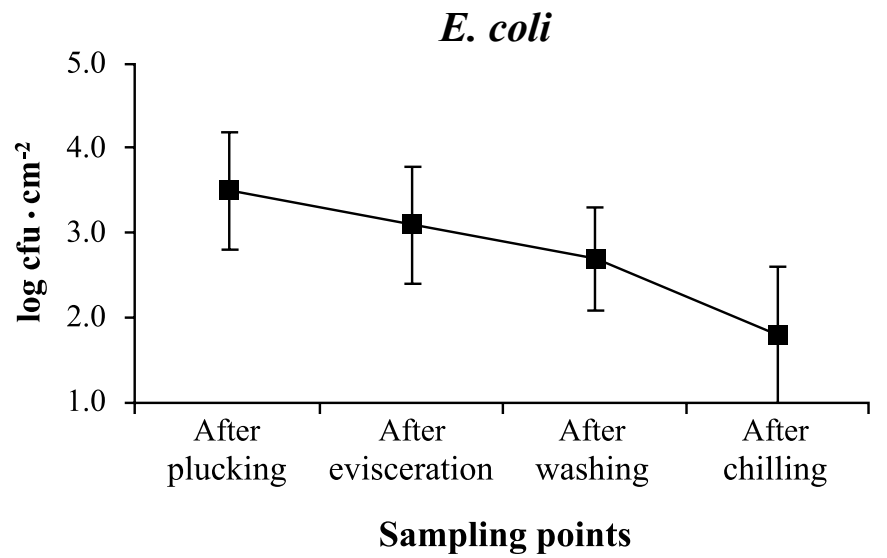

Fig. 2. Counts of $E$. coli (mean $\pm \mathrm{SD}$ ) on broiler carcasses during slaughter processing

A total of 14 strains of Listeria spp. were isolated from 12 carcasses $(7.5 \%)$. Pathogenic L. monocytogenes was not found while non pathogenic species $L$. welshimeri (9 strains) and L. innocua (3 strains) were detected; two cases were mixed cultures of the strains mentioned above. Listeria strains were found most frequently after plucking and evisceration ( 4 carcasses), 3 carcasses were found after washing and only 1 after chilling.

\section{Discussion}

Changes in contamination levels during poultry slaughter have been published by many authors. Mead (2004) reported a substantial decrease in TVC and coliform bacteria counts after carcass washing and after chilling. Lues et al. (2007) found that the highest counts of microorganisms were recorded in the initial stages of processing, comprising the receiving-killing and defeathering areas, whereas the counts toward the evisceration, air chilling, packaging and dispatch areas decreased. Ts ola et al. (2008) reported a significant $(P<0.001)$ decrease in both TVC, coliforms and E. coli after internal and external washing. In a Swedish study (Lindblat et al. 2006) TVC from 3.4 to $4.4 \log \mathrm{cfu} / \mathrm{cm}^{2}$ was recorded after chilling which is similar to our results. Lindblat et al. (2006) found about 1 log higher E. coli counts after chilling $\left(2.0-3.6 \log \mathrm{cfu} / \mathrm{cm}^{2}\right)$ than in our study. E. coli counts and TVC levels are comparable to those reported from, e.g., the United States where chlorine or other chemical decontaminants are used for decreasing of surface contamination. However, the use of those chemicals is not allowed in European Union member countries. The low level of contamination in the Czech Republic can be explained by good manufactory practice.

Salmonella enterica subsp. enterica is one of the major foodborne causes of gastroenteritis in most industrialised countries. The risk of poultry cross-contamination increases during transport; therefore, it is very important to thoroughly clean and disinfect transport crates immediately after the birds have been unloaded (Corry et al. 2002). Slaughtering broiler flocks colonized with Salmonella can lead to a contamination of both carcasses and the slaughter line. To reduce cross-contamination, it is necessary to apply proper slaughter logistics which means that flocks with a Salmonella-free status should be slaughtered first followed by Salmonella-positive flocks (Rasschaert et al. 2007). 
According to our findings, the investigated poultry carcasses were free of any significant contamination by Listeria spp. and especially L. monocytogenes during the slaughter process. Microbial contamination of the slaughterhouse premises was not, however, investigated in our study. A number of authors mention frequent findings of Listeria spp. both on poultry carcass surfaces and in the slaughterhouse itself, particularly at the end of the slaughter line (Rørvik et al. 2003). The authors hypothesize that the incidence of L. monocytogenes increases particularly during evisceration and in some other sections of the line, such as the packing or cutting of birds. Miettinen et al. (2001) assumed that increased contamination with $L$. monocytogenes occurs after chilling when carcasses are cut up and, mainly, when their skin is removed. Loura et al. (2004) reported frequent findings on poultry cuts and, especially, on the hands of slaughterhouse staff, and slaughterhouse desks and equipment. It follows from the above that Listeria spp. can persist in the slaughterhouse environment, and a high prevalence of these bacteria may be a sign of insufficient hygiene practices.

As was mentioned above, the microflora of poultry is very heterogeneous. Poultry meat contamination with microorganisms which cause deterioration in food quality, and especially those which cause foodborne diseases, is a major challenge for poultry industries in many countries that must aim at improving hygiene control during slaughter. In EU member states, principles of good manufacturing practice are used on farms and, for poultry slaughtering and processing, the HACCP system is the most important. Together with preventive measures on poultry farms and the use of modern slaughtering technologies, these systems can guarantee that poultry is produced with minimum microbial contamination and limited incidence of pathogens.

In our experiments we found that all the selected processing steps (plucking, evisceration, washing and chilling) decreased both the TVC and E. coli. In the case of TVC and $E$. coli the most effective processing step was washing. The numbers of Salmonella spp. and Listeria spp. were gradually lowered after each processing steps e.g. evisceration, washing and chilling. Research shows that selected processing steps during poultry slaughtering can decrease the TVC, E. coli, Salmonella spp. and Listeria spp.

\section{Acknowledgements}

The authors thank the team of poultry slaughterhouse workers for their cooperation. This study was funded by Project No. MSM 6215712402 of the Ministry of Education, Youth and Sports of the Czech Republic.

\section{References}

Berrang ME, Meinersmann RJ, Smith DP, Zhuang H 2008: The effect of chilling in cold air or ice water on the microbiological quality of broiler carcasses and the population of Campylobacter. Poultry Sci 87: 992-998

Blodgett R 2006: Appendix 2. Most probable number from serial dilutions. Bacteriological analytical manual online. Available at: www.cfsan.fda.gov/ ebam/bam-a2.html. Last modified October 2010. Accessed May 10, 2011

Bubert A, Koulet S, Goebel W 1992: The homologous and heterologous regions within the iap gene allow genusand species-specific identification of Listeria spp. by polymerase chain reaction. Appl Environ Microbiol 58: 2625-2632

Corry JEL, Allen VM, Hudson WR, Breslin MF, Davies RH 2002: Sources of salmonella on broiler carcasses during transportation and processing: modes of contamination and methods of control. J Appl Microbiol 92: 424-432

Cox JM, Pavic A 2010: Advances in enteropathogen control in poultry production. J Appl Microbiol 108: 745-755

Huang B, Eglezos S, Heron BA, Smith H, Graham T, Bates J, Savill J 2007: Comparison of multiplex PCR with conventional biochemical methods for the identification of Listeria spp. isolates from food and clinical samples in Qeensland, Australia. J Food Prot 70: 1874-1880

James C, Vincent T, Andrade Lime TI, James SJ 2006: The primary chilling of poultry carcasses-a review. Int J Refriger 29: 847-862

Keener KM, Bashor MP, Curtis PA, Sheldon BW, Kathariou S 2004: Comprehensive review of Campylobacter and poultry processing. Compr Rev Food Sci Food Safety 3: 105-116 
Lindblad M, Lindmark H, Thiested Lamberetz S, Lindqvist R 2006: Microbiological baseline study of broiler chickens at Swedish slaughterhouses. J Food Prot 69: 2875-2882

Loura CAC, Almeida RCC, Almeida PF 2004: The incidence and level of Listeria spp. and Listeria monocytogenes contamination in processed poultry at a poultry processing plant. J Food Safety 25: 19-25

Lues JF, Theron MM, Venter P, Rasephei MH 2007: Microbial composition in bioaerosols of a high-throughput chicken-slaughtering facility. Poult Sci 86: 142-149

Mead GC 2004: Microbial hazards in production and processing. In Mead GC (Ed.): Poultry meat processing and quality. Woodhead Publishing, Cambridge, pp. 232-257

Miettinen MK, Palmu L, Bjorkroth KJ, Korkeala H 2001: Prevalence of Listeria monocytogenes in broilers at the abattoir, processing plant and retail level. J Food Prot 64: 994-999

Rasschaert G, Houf K, De Zutter L 2007: Impact of the slaughter line contamination on the presence of Salmonella on broiler carcasses. J Appl Microbiol 103: 333-341

Rørvik LM, Aase B, Alvestad T, Caugant DA 2003: Molecular epidemiological survey of Listeria monocytogenes in broilers and poultry products. J Appl Microbiol 94: 633-640

Thomas NL 1978: Observations of the relationship between the surface area and weight of eviscerated carcasses of chickens, ducks and turkeys. J Food Technol 13: 81-86

Tsola E, Drosinos EH, Zoiopoulos P 2008: Impact of poultry slaughter house modernisation and updating of food safety management systems on the microbiological quality and safety of products. Food Control 19: 423-431 